\title{
Diagnostic prediction of ovine fracture healing outcomes via a novel multi-location direct electromagnetic coupling antenna
}

\author{
Jakob G. Wolynski ${ }^{1} \wedge$, Kevin M. Labus ${ }^{1}$, Jeremiah T. Easley ${ }^{2}$, Branislav M. Notaroš $^{3}$, Milan M. Ilic ${ }^{4} \wedge$, \\ Christian M. Puttlitz ${ }^{1}$, Kirk C. McGilvray ${ }^{1 \wedge}$
}

${ }^{1}$ Orthopaedic Bioengineering Research Laboratory, Departments of Mechanical Engineering and School of Biomedical Engineering, Colorado State University, Fort Collins, CO, USA; ${ }^{2}$ Preclinical Surgical Research Laboratory, Department of Clinical Sciences, Colorado State University, Fort Collins, CO, USA; ${ }^{3}$ Department of Electrical and Computer Engineering, Colorado State University, Fort Collins, CO, USA; ${ }^{4}$ School of Electrical Engineering, University of Belgrade, Belgrade, Serbia

Contributions: (I) Conception and design: All authors; (II) Administrative support: JT Easley, CM Puttlitz, KC McGilvray; (III) Provision of study materials or patients: JG Wolynski, JT Easley; (IV) Collection and assembly of data: JG Wolynski; (V) Data analysis and interpretation: JG Wolynski, KM Labus, CM Puttlitz, KC McGilvray; (VI) Manuscript writing: All authors; (VII) Final approval of manuscript: All authors.

Correspondence to: Kirk C. McGilvray, PhD. 1374 Campus Delivery, Fort Collins, CO 80523, USA. Email: kirk.mcgilvray@colostate.edu.

\begin{abstract}
Background: Expedient prediction of adverse bone fracture healing (delayed- or non-union) is necessary to advise secondary treatments for improving healing outcome to minimize patient suffering. Radiographic imaging, the current standard diagnostic, remains largely ineffective at predicting nonunions during the early stages of fracture healing resulting in mean nonunion diagnosis times exceeding six months. Thus, there remains a clinical deficit necessitating improved diagnostic techniques. It was hypothesized that adverse fracture healing expresses impaired biological progression at the fracture site, thus resulting in reduced temporal progression of fracture site stiffness which may be quantified prior to the appearance of radiographic indicators of fracture healing (i.e., calcified tissue).
\end{abstract}

Methods: A novel multi-location direct electromagnetic coupling antenna was developed to diagnose relative changes in the stiffness of fractures treated by metallic orthopaedic hardware. The efficacy of this diagnostic was evaluated during fracture healing simulated by progressive destabilization of cadaveric ovine metatarsals treated by locking plate fixation $(\mathrm{n}=8)$. An ovine in vivo comparative fracture study $(\mathrm{n}=8)$ was then utilized to better characterize the performance of the developed diagnostic in a clinically translatable setting. In vivo measurements using the developed diagnostic were compared to weekly radiographic images and postmortem biomechanical, histological, and micro computed tomography analyses.

Results: For all cadaveric samples, the novel direct electromagnetic coupling antenna displayed significant differences at the fracture site $(\mathrm{P}<0.05)$ when measuring a fully fractured sample versus partially intact and fully intact fracture states. In subsequent in vivo fracture models, this technology detected significant differences $(\mathrm{P}<0.001)$ in fractures trending towards delayed healing during the first 30 days post-fracture.

Conclusions: This technology, relative to traditional X-ray imaging, exhibits potential to greatly expedite clinical diagnosis of fracture nonunion, thus warranting additional technological development.

Keywords: Fracture healing; bone; nonunion; diagnostic; ovine

Submitted Apr 24, 2021. Accepted for publication Jun 23, 2021.

doi: 10.21037/atm-21-1853

View this article at: https://dx.doi.org/10.21037/atm-21-1853

^ ORCID: Jakob G. Wolynski, 0000-0003-2659-337X; Milan M. Ilić, 0000-0003-4196-3594; Kirk C. McGilvray, 0000-0003-1302-9307. 


\section{Introduction}

Failed bone fracture healing (nonunion) yields substantially harmful effects on patients' wellbeing including the need for additional orthopaedic surgeries, increased duration of outpatient physical therapy, onset of chronic pain, extended opioid therapy duration, increased likelihood of strong opioid prescription, and $118 \%$ increase in reported treatment costs $(1,2)$. Reported rates of nonunion vary according to fracture location, severity, treatment type, and patient-specific risk factors; however, nonunions are especially prevalent among long bone fractures such as the tibia where up to $12 \%$ of fractures are reported to experience nonunion (1). Early diagnosis of aberrant fracture healing is imperative as early intervention will allow clinicians to implement adjunct therapies much earlier in the healing cascade to meaningfully reduce patient suffering and the associated economic burden $(3,4)$.

Bone is an exceptionally resilient tissue owing to its ability to repair without the formation of chronic scar tissues. Fracture healing is generally characterized by overlapping phases of inflammation, repair, and remodeling $(5,6)$. The inflammatory phase begins at the onset of bone fracture which causes blood vessel rupture ultimately leading to the formation of a hematoma at the fracture site $(5,7)$. Following inflammation, fracture repair occurs through endochondral and/or intramembranous ossification, depending on the mechanical environment of the fracture site (5) which is influenced by clinical fixation (8) and rehabilitation techniques (9). Intramembranous ossification is characterized by direct formation of woven bone to bridge the fracture gap, and tends to be limited to rigidly stabilized reduced fractures (10) experiencing small magnitudes of strain (11) and interfragmentary motion (5). Alternatively, endochondral ossification occurs when the fracture site is subjected to moderate strains (11) and interfragmentary motion (5). Endochondral ossification involves the formation of intermediate soft callus tissues, composed of cartilaginous and granular tissues, which are subsequently resorbed after becoming calcified (12). Fracture nonunion occurs when a cessation of this biological progression occurs prior to successful boney bridging of the fracture gap (13), and can result from infection or deficiency in the mechanical or biological environment of the fracture site (14-16). The complex biomechanical regulation of fracture healing $(17,18)$ and numerous patient comorbidities which may inhibit fracturing healing (19) makes clinical prediction of fracture repair outcome an arduous task.
Clinical diagnosis of nonunion remains a slow process, requiring on average 6.2 months to diagnose with current clinical approaches (20). Much of this delay can be attributed to the standard diagnostic use of planar X-ray imaging which remains highly qualitative and subjective (20-24), thus increasing the difficulty to predict fracture union state during the early stages of healing ( $<30$ days). This results in a $50 \%$ probability of $\mathrm{X}$-ray imaging correctly diagnosing the correct stage of fracture healing (25) and facilitates restricted ability to forecast the occurrence of delayed- and non-unions $(26,27)$. Furthermore, $\mathrm{X}$-ray imaging has exhibited poor performance as a means to predict the mechanical stiffness of the healing fracture $(23,26,28)$, which is a vital metric for patients' safe weightbearing and return to normal daily activity. Fracture stiffness is often addressed by manual manipulation of the fracture site to qualitatively determine fracture stability; however, this technique remains subjective and thus is limited by clinician interpretation (29).

Despite the inability to be quantified by X-ray imaging, fracture stiffness shows promise as an effective means to diagnose and predict fracture healing outcome (13,30-33). Previous studies have shown that mechanical load is temporally shifted from orthopaedic fixation hardware (i.e., plates, intramedullary rods, or external fixation) to the bone $(13,30-35)$ as healing progresses. Due to morphologic changes at the fracture site (i.e., the formation and remodeling of the fracture callus) which precede tissue mineralization, quantification of fracture site stiffness has shown the ability to accurately predict healing outcome prior to radiographic diagnosis $(31,32)$. Studies have also shown that during acute healing ( $<30$ days) fractures progressing to proper union will exhibit progressive improvement of mechanical strength, while this progression of increased fracture site stiffness is arrested and/or delayed in fractures trending towards nonunion (31).

While biomechanical quantification of fracture site stiffness has shown capacity as an early diagnostic of fracture healing outcome, there are currently no noninvasive techniques to perform this measurement in a clinical setting. Previous research groups have performed this analysis using wired strain gages on orthopaedic plates $(32,34)$, instrumented external fixators (30,35-39), and plates instrumented with telemetric sensors (40); however, these techniques are respectively limited by the potential for infection, limited hardware application, and longterm biocompatibility concerns. To address this clinical deficit, our research group previously developed wireless 
implantable microelectromechanical sensors (bioMEMS) capable of quantifying, via external antenna, temporal changes to the mechanical strain on implanted orthopaedic hardware $(31,41-49)$. In a previous study, temporally repeated bioMEMS measurements detected statistically significant differences in normal and aberrant bone healing in an ovine fracture model in as few as 21 days postfracture (31), and use of flexible bioMEMS enabled sensor application to most types of orthopaedic hardware (49). Despite the demonstrated promise of these bioMEMS technologies, clinical translation of these sensors remains challenging as their implementation required implant modification to attach and protect the sensors during surgical implantation. These implant modifications, while minor, resulted in associated additional regulatory hurdles to achieve clinical applicability.

To address these limitations, our research group has developed a diagnostic system capable of measuring temporal changes in the relative fracture callus stiffness for any fracture treated with standard off-the-shelf orthopaedic hardware stabilization devices, without the need to modify existing hardware design (50). This diagnostic relies upon fractures with orthopaedic implant fixation behaving as a composite structure, where stiffness of the healing fracture tissues and the implanted hardware both contribute to the stiffness of the composite structure. Since it can be assumed that the implant's stiffness remains constant during healing, changes to the stiffness of the healing fracture site yields quantifiable changes to the stiffness of the boneimplant composite. Our research group has developed a diagnostic device which quantifies this stiffness by applying a physiologic non-detrimental mechanical load to the fracture site (i.e., stabilized bone) while measuring the induced deflection of the fixation hardware via direct electromagnetic coupling (DEC) with an external antenna (51). Succinctly, the external telemetric DEC antenna electromagnetically couples with the implanted hardware positioned in its near-field region, resulting in an apparent resonant frequency (ARF, in the $\mathrm{MHz}$ range) which shifts with changing distance between the coupled members. Thus, measuring ARF shifts during controlled mechanical loading provides a non-invasive means to indirectly measure the stiffness of a fracture treated by any existing metallic orthopaedic implant. Theoretically, temporally repeated DEC measurements can thus indicate if proper healing progression is occurring, as indicated by temporally increasing relative fracture stiffness, or if healing progression has slowed or stopped, as indicated by invariant fracture stiffness prior to fracture union.

We hypothesized that DEC would enable early diagnosis and prediction of aberrant fracture healing by detecting differences in relative fracture stiffness progression during the first 30 days of the healing cascade. In this study we developed a DEC antenna array capable of measuring the displacement of orthopaedic hardware at multiple discrete locations to better characterize the mechanical stiffness of a healing long bone fracture. The efficacy of this diagnostic device was first tested by in vitro simulation of a healing long bone fracture treated by orthopaedic plating. These experiments culminated in an in vivo large animal fracture healing model in which the DEC diagnostic device was tested on critical and non-critical ovine fractures treated by orthopaedic plating.

The following article is presented following the ARRIVE reporting checklist (available at https://dx.doi.org/10.21037/ atm-21-1853).

\section{Methods}

\section{Study design}

The purpose of this study was to develop a novel technology to diagnose and predict bone fracture healing outcome. The efficacy of the technology was initially evaluated in fracture healing simulated by the progressive destabilization of cadaveric ovine metatarsals. This experiment served to provide initial validation of the DEC technology while minimizing unnecessary loss of life for in vivo experimentation. Tissues for this cadaveric experiment were obtained from animals sacrificed for unrelated studies. The results of the initial cadaveric experiments justified progression to an in vivo comparative fracture model where the efficacy of the DEC technology in a more clinically translatable setting could be evaluated. A pilot study was performed to provide initial data for power analysis to determine the appropriate sample size such that sufficient statistical power could be achieved while minimizing animal use. Experiments performed by this study were approved by the Colorado State University Institutional Animal Care and Use Committee (CSU IACUC approval \#198990A). Two fracture models ( $\mathrm{n}=4$ animals per group) were implemented to simulate proper or delayed fracture healing according to the size of the surgically induced osteotomy. The animals were evenly and randomly divided between the two fracture models by an individual who was impartial and blinded to the study. Power analysis of the 


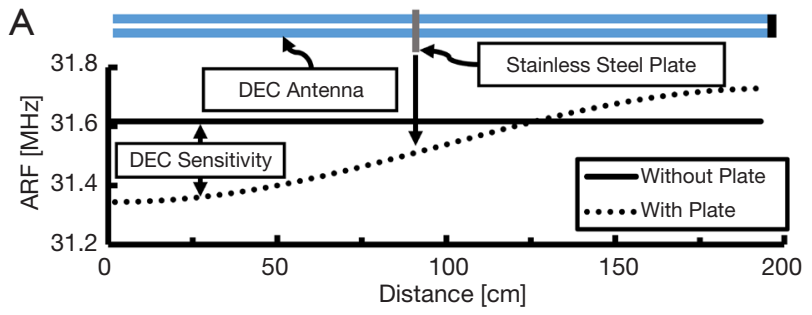

B
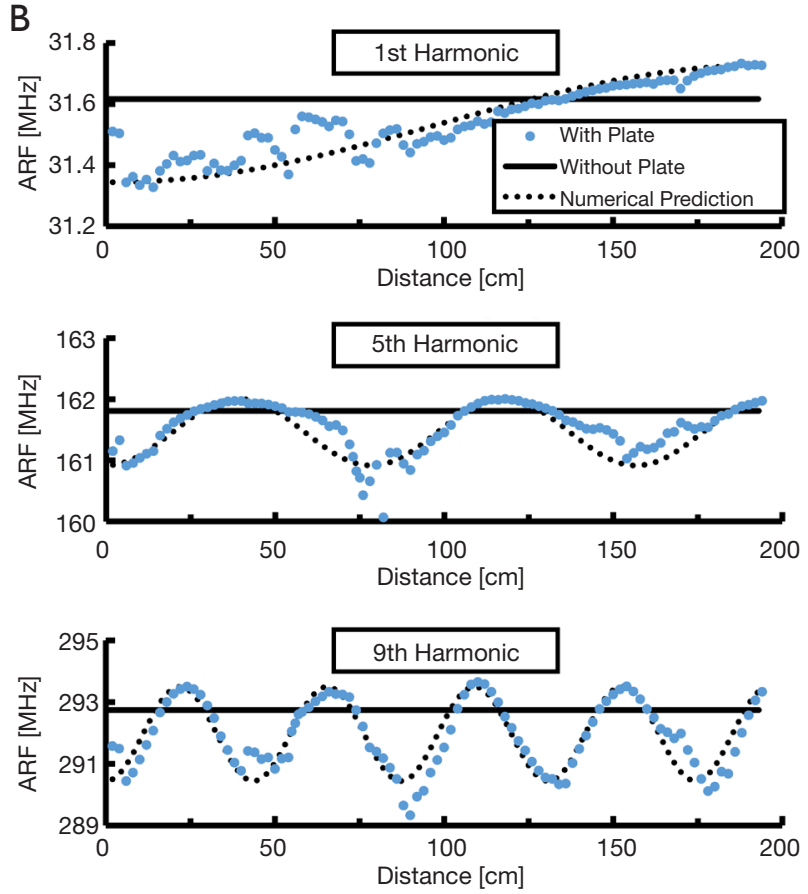

Figure 1 Direct electromagnetic coupling (DEC) sensitivity as a function of antenna location. (A) Experimental setup to determine dipole antenna sensitivity to orthopaedic plate proximity at various locations along the antenna's length. DEC sensitivity is found as the difference between the apparent resonant frequencies (ARFs) measured with and without the plate at a given location along the antenna's length. (B) Experimental results for the first, fifth, and ninth ARF harmonics. Each harmonic illustrates a quantity of locations of maximum DEC sensitivity equal to (harmonic number $+1) / 2$. Dashed line represents a numerical prediction of ARF defined by Eq. [1].

primary data of interest, the rate of change of DEC antenna sensitivity during early fracture healing, indicated the initial sample size was sufficient to produce statistically significant differences $(\mathrm{P}<0.05)$ with a power value exceeding 0.99 ; thus, no additional animals were included in this study. DEC sensitivity values were measured weekly throughout healing, with five replicate data collections performed and averaged within each testing session to minimize the occurrence of outlier data points. Experimentation was not performed in a blinded fashion due to readily apparent visual differences in the treated limbs indicating differences in fracture model. Environmental confounders were not controlled. Experimental data points were only removed in the event of gross experimental error.

\section{DEC antenna sensitivity to orthopaedic implant deflections}

A dipole antenna was created by aligning two identical 2 meter coaxial cables (141-2MSM+; Mini-Circuits; Brooklyn, NY, USA) and electrically connecting their shielding elements near their connectors. The coaxial cables were placed parallel to one another, with approximately $3 \mathrm{~mm}$ separation, in a straight line along a non-conductive surface. The end of one cable was connected to a vector network analyzer (VNA) (TTR500; Tektronix; Beaverton, OR, USA) by a coaxial lead cable (Micro-Coax UTIFLEX; CarlisleIT; Pottstown, PA, USA) which measured the reflection coefficient, defined as the ratio of power returned and power supplied to that port $\left(\mathrm{S}_{11}, \mathrm{~dB}\right)$, over a range of electromagnetic frequencies $(10-500 \mathrm{MHz})$. ARF was measured as the frequency at which $S_{11}$ minima occurred; ARF occurred harmonically at increasing frequencies. The baseline ARF of a given harmonic was measured prior to introducing a stainless steel plate $(120 \mathrm{~mm} \times 20 \mathrm{~mm} \times$ $10 \mathrm{~mm}$ ) on the free end of the dipole antenna (away from the connection to the coaxial lead cable). The ARF was then measured as the plate was moved along the length of the antenna in $2 \mathrm{~mm}$ increments. Antenna locations of maximum DEC sensitivity were determined to be the sites where introduction of the plate resulted in the largest difference in ARF, relative to the baseline ARF (Figure 1A,1B). This analysis was repeated for the first 11 odd antenna harmonics. The cable was then coiled to align three locations of maximum DEC sensitivity to improve sensitivity in a focused area (51), creating a coiled antenna construct (Figure 2A).

\section{DEC antenna array sensitivity to cross-talk}

Simultaneous DEC measurement at multiple discrete locations may provide clinically useful data. For example, multi-location measurements may provide useful information in cases of complex fracture types where multiple orthopaedic implants may be utilized on the same bone. To create a diagnostic antenna capable of measuring 

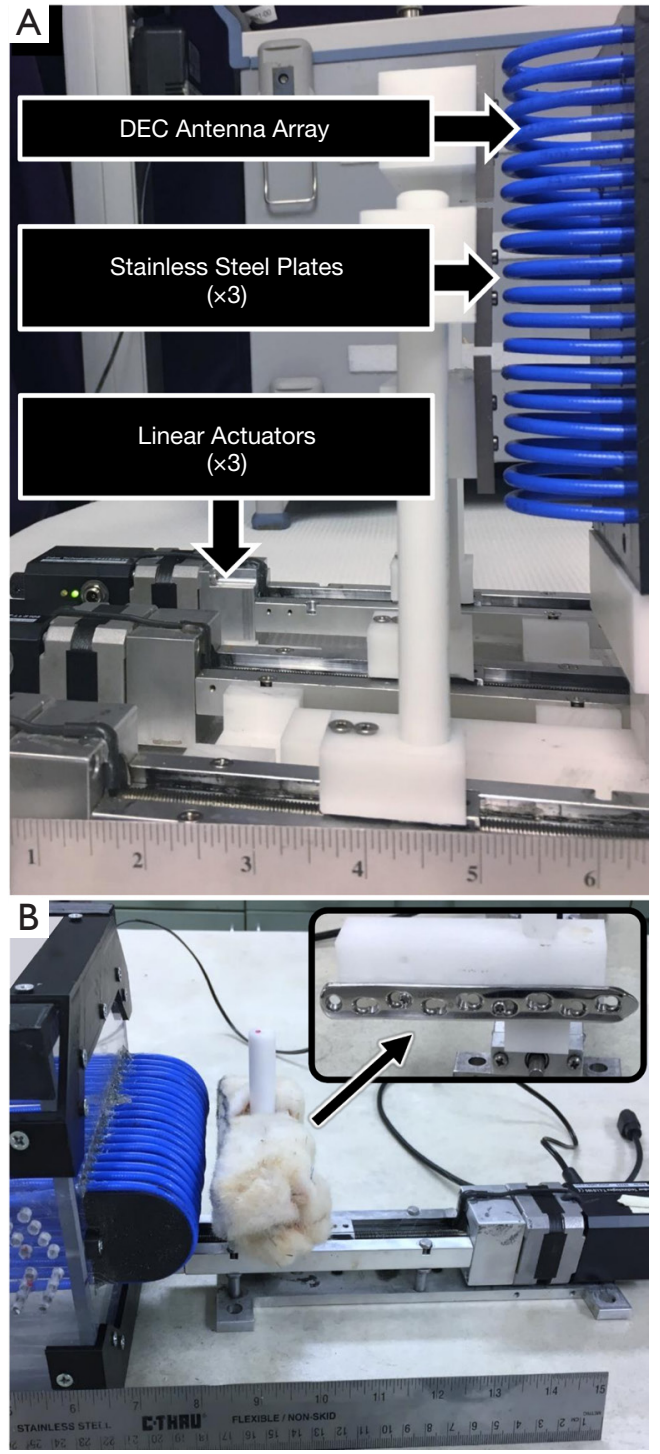

Figure 2 Multi location direct electromagnetic coupling (DEC) antenna array sensitivity at discrete locations. (A) Experimental setup in which linear actuators were used to increase plate-antenna displacement at a single antenna location (i.e., one third of the antenna array) while interrogating the response from all three antenna locations. (B) Experimental setup to evaluate antennnae sensitivity to orthopaedic plate displacements, as produced by precision linear actuators, in the presence of physiologic tissues.

in multiple discrete regions, it was necessary to produce an array of multiple antennas. The array was selected to feature three individual antennas to facilitate discrete DEC measurements of the proximal, midspan, and distal thirds on a long bone. Three antennas were produced and stacked so that their DEC regions of sensitivity aligned linearly. A series of experiments were performed to select the antenna designs, including measurement harmonic numbers, so that changes to the DEC coupling distance in one antenna would elicit little to no effect in the ARF of the other antennas (i.e., cross-talk). A custom testing fixture was designed to enable three stainless steel plates, of combined dimensions comparable to one orthopaedic fixation plate $(40 \mathrm{~mm} \times 20 \mathrm{~mm} \times 10 \mathrm{~mm}$, per plate), to be individually displaced by precision linear actuators (T-LLS105; Zaber Technologies; Vancouver, BC, Canada) to independently alter the DEC coupling distance of each antenna in the array (Figure 2A). The distance between each plate and antenna were sequentially increased (0.0-2.0 $\mathrm{mm}, 0.2 \mathrm{~mm}$ increments) while measuring the ARF of each antenna in the array. ARF of the proximal and distal antennas were measured using S11 and S22 measurements, respectively, from a two port VNA (R\&S ZVB4; Rhode \& Schwarz; Munich, Germany; 1,000 linearly spaced frequency measurement points spanning $20 \mathrm{MHz}$ window centered upon initial ARF; $10 \mathrm{dBm}$ supplied power) while ARF was measured via S11 of a second VNA (Tektronix TTR500; 500 linearly spaced frequency measurement points spanning $10 \mathrm{MHz}$ window centered upon initial ARF; $10 \mathrm{dBm}$ supplied power). Experiments were conducted with parametrically differing designs for each antenna until a design exhibited minimal change in antenna ARF when the DEC coupling distance was changed for an adjacent antenna (i.e., the ARF of each antenna would only change if the coupling distance was changed for that specific antenna). A set of antennas designed for measurement at the $25^{\text {th }}$ harmonic exhibited this behavior and were utilized for more thorough follow up tests.

\section{DEC antenna array sensitivity to orthopaedic plates}

To further investigate the sensitivity of the final DEC antenna array, an orthopaedic locking compression plate (LCP; VP4045.09; DePuy Synthes; Warsaw, IN, USA) was fixed to a precision linear actuator. Cadaveric ovine skin was sutured over the LCP to replicate the plated ovine metatarsal used in subsequent in vitro and in vivo studies. The plate was centered along the length of the antenna array so that each individual antenna measured approximately $1 / 3$ of the orthopaedic plate (Figure $2 B$ ). The plate was incrementally displaced $(0-20 \mathrm{~mm}, 0.01 \mathrm{~mm}$ step size) from the array as the ARF from each antenna was 

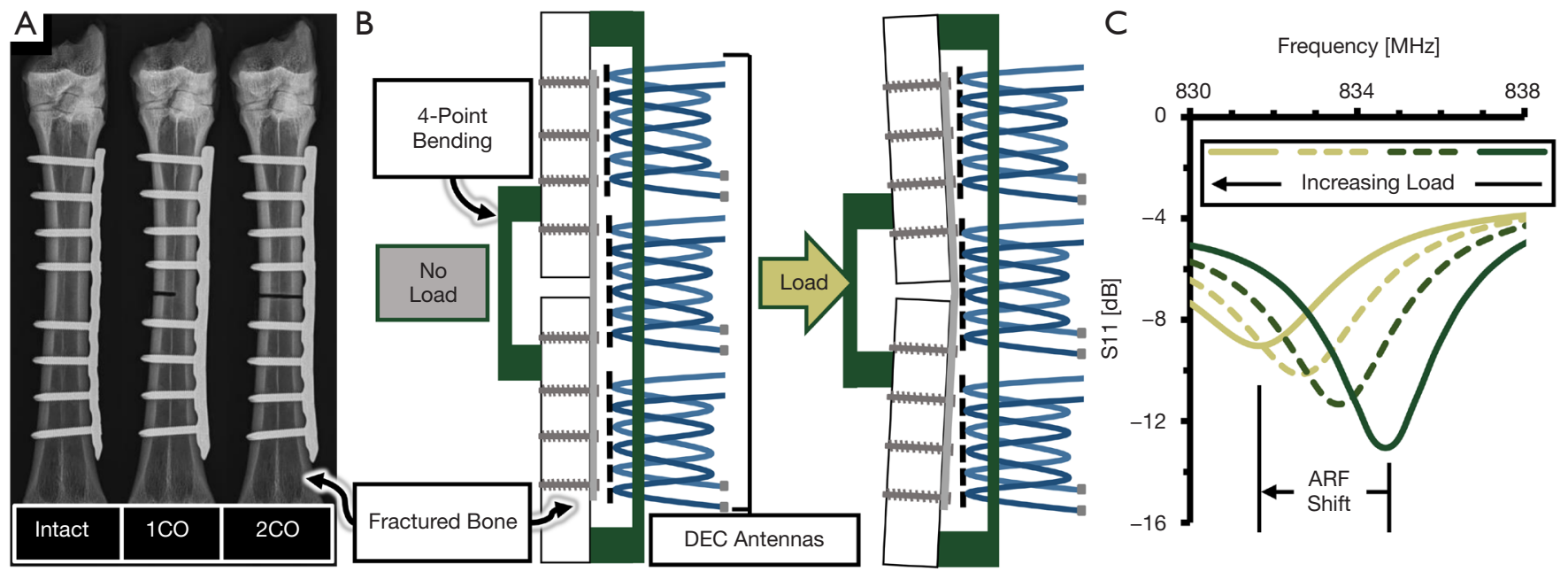

Figure 3 Benchtop evaluation of direct electromagnetic coupling (DEC) sensitivity to changes in fracture stiffness. (A) Different stages of fracture healing were simulated by progressively destabilizing the mid-diaphysis of a plated ovine metatarsal via increasing depth of bone saw cuts. Each fracture state was loaded in the custom loading fixture (1.0-2.0 N-m in $0.25 \mathrm{~N}-\mathrm{m}$ increments, $\mathrm{n}=5$ loading cycles per data collection, $\mathrm{n}=8$ total cadaveric samples) while recording DEC antenna responses. (B) Graphical representation of the custom four-point bending loading fixture used to produce deflections of orthopaedic hardware towards the developed multi-location DEC antenna array. (C) Using this device to apply load to a bone treated by plate fixation causes the plate-antenna distance to change resulting in quantifiable shifts in apparent resonant frequency (ARF) proportionate to the amount of plate deflection.

measured. For all data collection henceforth, ARF of the proximal and distal antennas were measured using S11 and S22 measurements, respectively, from a two port VNA (R\&S ZVB4; response measured from 805-825 MHz via 1,000 linearly spaced points; $10 \mathrm{dBm}$ supplied power) while ARF was measured via S11 of a second VNA (TTR500; response measured from $830-840 \mathrm{MHz}$ via 500 linearly spaced points; $10 \mathrm{dBm}$ supplied power). ARF values were collected using a custom data acquisition program (LabVIEW 2018; National Instruments; Austin, TX, USA).

\section{DEC differences in cadaveric fractures of varying stiffness}

A custom DEC-loading fixture was designed to enable the application of mechanical four-point bending to a long bone with orthopaedic hardware fixation, thus inducing implant deflection towards the DEC antenna array discussed in the previous section (Figure 3). Four-point bending was selected for this application as it allows the fracture site to be placed between the inner bending points to avoid direct contact of the trauma site. Additionally, this loading mechanism produces a constant bending moment across the fracture site with minimal direct contact forces due to inherent mechanical advantage of the bending device. The bending moment was induced by modulating the contact force of the pneumatically actuated inner bending contact points. Contact forces were measured via load cell (Model 53; Honeywell; Charlotte, NC, USA) and converted to maximum bending moment according to the fixture geometry.

Cadaveric ovine metatarsals were obtained from the hind limbs of animals $(n=8)$ sacrificed for unrelated studies. A 316L stainless steel 9-hole locking compression plate (LCP; VP4045.09; DePuy Synthes; Warsaw, IN, USA) was surgically implanted on the lateral midspan of the metatarsal and secured with eight locking screws (24 mm length) with the middle hole left empty to facilitate eventual osteotomy at this location. The plated metatarsal was then loaded into the DEC-loading device with the LCP centered between the outer bending points and aligned along the length of the DEC antennas (Figure 3B). For all tests, the metatarsal was placed such that the distance between the antennas and the surface of the skin was less than $1 \mathrm{~mm}$ at the proximal and distal aspects of the antenna array. Four-point bending loads were applied $(1.0-2.0 \mathrm{~N}-\mathrm{m}$ in $0.25 \mathrm{~N}-\mathrm{m}$ increments, $\mathrm{n}=5$ loading cycles per data collection) while measuring antenna ARF. Following loading of the intact metatarsal, the sample was removed from the loading fixture and a bone saw was 
used to destabilize the mid-diaphyseal cortex contralateral to the LCP. Monocortical (1CO) and bicortical (2CO) fracture states were induced by progressively removing bone, with each fracture state receiving four-point bending and DEC evaluations (Figure 3). The antenna sensitivity, a relative and inverse measure of the fracture's mechanical stiffness, was calculated as the slope of the resultant ARF/ load data. Mean antenna sensitivities were calculated for each antenna and fracture state by averaging the sensitivities of all individual loading cycles.

\section{In vivo DEC temporal progression of proper and aberrant bealing fractures}

Experiments were performed under a project license (No.: 19-8990A) granted by the Colorado State University Institutional Animal Care and Use Committee, in compliance with United States and state of Colorado guidelines for the care and use of animals. Eight skeletally mature sheep (Rambouillet cross, female, $>3$ years of age) were utilized for this study, and housed in Colorado State University Veterinary Teaching Hospital facilities for the duration of the study. Sheep were selected for this experimental model due to their similarity to humans in several facets pertinent to the study of bone healing: body weight, bone macrostructure (52), bone mineral composition, cortical bone microarchitecture, and bone healing rates (53). The animals were anesthetized and the fracture site was sterilized according to standard surgical practices. A 10 -cm skin incision was made over the lateral aspect of the metatarsal. Overlying soft tissue and periosteum were elevated from the bone. A 9-hole LCP (VP4045.09; DePuy Synthes; Warsaw, IN, USA) was contoured to the metatarsal and the required holes were drilled under saline irrigation. An osteotomy was created at the metatarsal midspan by oscillating surgical saw under saline irrigation. Proper union (PU) and delayed union (DU) surgical models were created by producing a $3-\mathrm{mm}$ or $6-\mathrm{mm}$ osteotomy, respectively, according to results from a previous pilot study. The animals were evenly and randomly divided between the two fracture models. The plate was then attached to the bone using four bicortical locking screws in each bone fragment with the middle screw hole centered over the osteotomy gap. The middle screw hole was filled with the head of a locking screw in order to reduce the risk of plate failure (Figure 4A) resulting from the large stressriser of an unfilled screw hole at the fracture site. The surgical site was closed using standard suturing techniques.
The animals received immediate hard casting on the treated limb and were allowed to freely ambulate. The hard cast was bi-valued to facilitate temporary removal for DEC testing and radiographic imaging during the initial stages of healing, and was removed permanently after 3 -weeks postsurgery.

DEC evaluations were preformed twice-weekly during the initial 3-weeks post-surgery, and then weekly until animal euthanasia. During DEC evaluations animals were placed under general anesthesia and positioned in lateral recumbency to minimize animal discomfort and prevent limb movement from confounding DEC measurements of orthopaedic plate displacement. The DEC-loading fixture was utilized to apply four-point bending loads (1.0-2.0 N-m in $0.25 \mathrm{~N}-\mathrm{m}$ increments for $\mathrm{n}=5$ tests) to the treated limb while measuring the resultant ARF shifts (Figure 4B). For all tests, the metatarsal was positioned such that the distance between the DEC antennas and the surface of the skin was less than $1 \mathrm{~mm}$ at the proximal and distal aspects of the antenna array. Mean antenna sensitivities were calculated according to the same methods as the aforementioned in vitro experiments. To account for inter-specimen differences in initial limb stiffness resulting from intrinsic variations in anatomic bone structure, normalized antenna sensitivities were quantified as a percentage of each specimen's maximum antenna sensitivity exhibited throughout the study duration. The use of these data as a diagnostic metric was driven by the presumption that relative changes, rather than the absolute value, of fracture stiffness indicate whether fractures are progressing towards proper or adverse healing outcomes. The use of DEC antenna sensitivity as a diagnostic metric was thus supported by the results of the prior benchtop and ex vivo experiments which indicated DEC antenna sensitivity to vary with changes in fracture stiffness. Antenna sensitivity rate of change during the initial stages of healing was calculated as the slope of a linear fit applied to the normalized antenna sensitivities during the first 30 days post fracture (31). Weekly bi-planar radiographic images of the treated limbs were taken throughout the duration of the study.

Animals from the union and delayed-union surgical models were euthanized at 8- and 10-week post-surgery, respectively. Following euthanasia, the metatarsals were immediately collected $(\leq 30 \mathrm{~min})$ and soft tissue was removed. The bending stiffness of each sample was measured by four-point bending in a servo-hydraulic testing system (858 MiniBionix; MTS Systems Corp; Eden Prairie, $\mathrm{MN}$, USA). Following five preconditioning cycles, samples 


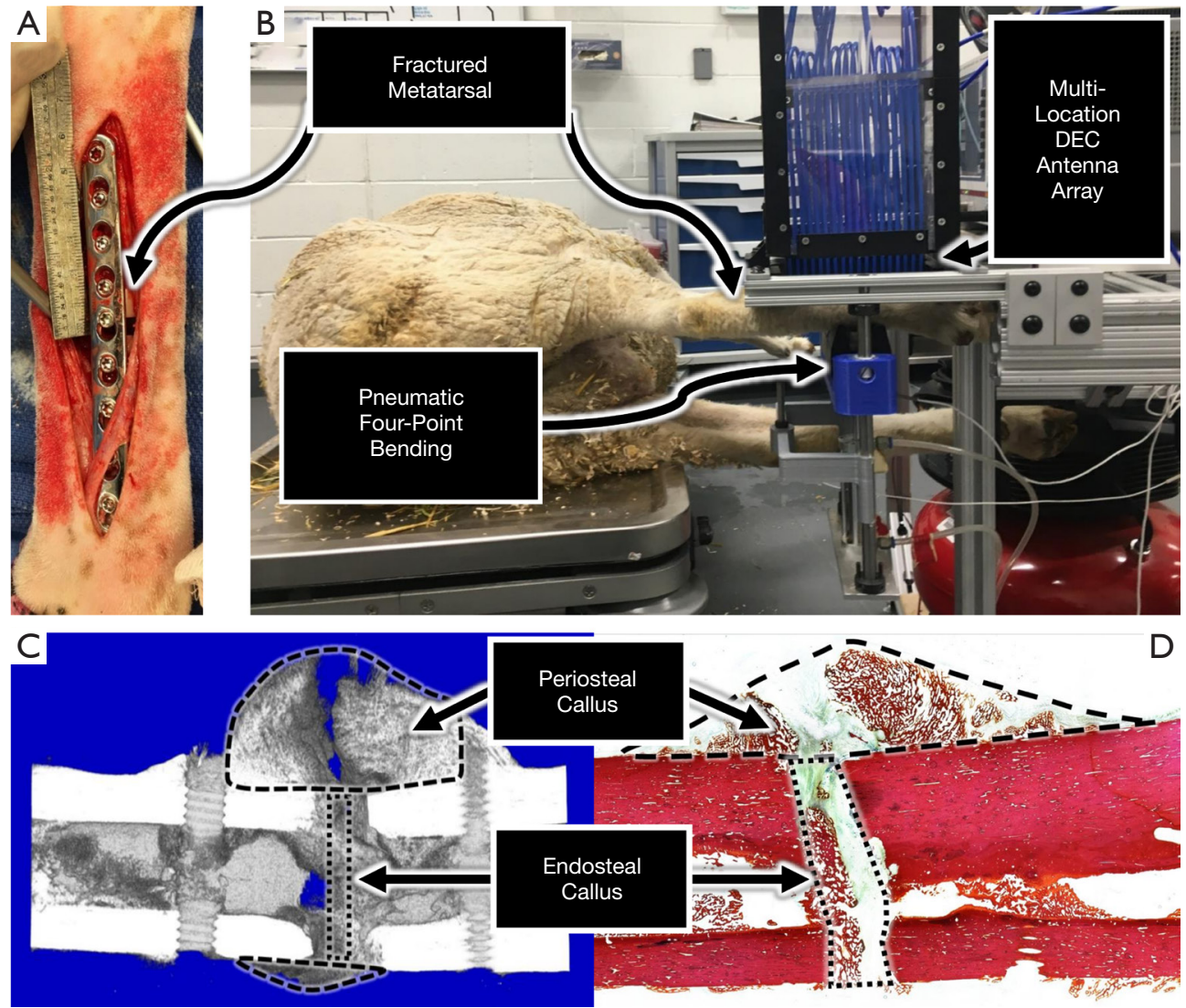

Figure 4 Direct electromagnetic coupling (DEC) diagnostic data in an ovine comparative fracture model. (A) Non-reduced ovine middiaphyseal fractures were produced by surgical osteotomy of large $(5.95 \pm 1.32 \mathrm{~mm}, \mathrm{n}=4$ specimen $)$ or small $(2.80 \pm 1.15 \mathrm{~mm}, \mathrm{n}=4 \mathrm{specimen})$ heights in order to produce delayed union (DU) or proper union (PU) healing outcomes, respectively. (B) DEC measurements of relative fracture stiffness were obtained by applying four-point bending loads (1.0-2.0 N-m in $0.25 \mathrm{~N}-\mathrm{m}$ increments for $\mathrm{n}=5$ tests) while recording the resultant apparent resonant frequency (ARF) shifts in each DEC antenna. (C,D) Postmortem analysis was performed to compare, for the proper union and delayed union fracture models, tissue composition in the periosteal and endosteal callus regions of interest (ROI) using (C) $\mu \mathrm{CT}$ analysis and (D) mid-transverse histological section stained by with Sanderson's Rapid Bone Stain and counter stained with Van Gieson.

received an additional five loading cycles of cranial-caudal bending $(0.5-3.0 \mathrm{~N}-\mathrm{m}, 0.05 \mathrm{~mm} / \mathrm{s}$ crosshead displacement rate). Bending stiffness was calculated from the slope of the linear portion of the moment-displacement data. Following initial bending tests, the LCP and hardware were removed and testing was repeated for the hardware-free bone.

Following biomechanical testing, the samples were placed in $70 \%$ ethanol solution. Micro-computed tomography $(\mu \mathrm{CT})$ analyses were performed at the fracture site (voltage: $70 \mathrm{kVp}$, current: $114 \mu \mathrm{A}$, integration time: $500 \mathrm{~ms}$; Scanco $\mu \mathrm{CT}$ 80; Scanco Medical AG; Bruttisellen, Switzerland). Three dimensional images were rendered from the $\mu \mathrm{CT}$ data and used to produce mid-transverse slices to investigate cortical bone bridging across the fracture site. Total bone volume $(\mathrm{TBV})$, bone volume fraction $(\mathrm{BVF}=\mathrm{TBV} /$ total volume), and bone mineral density $\left(\mathrm{BMD}=\mathrm{mg} \mathrm{HA} / \mathrm{cm}^{3}\right)$ were quantified for the endosteal callus region of interest (ROI) consisting of a $1.5 \mathrm{~mm}$ thick section at the fracture midspan, and for the periosteal fracture callus ROI defined as the section between the screw holes adjacent to the fracture, excluding the diaphyseal bone (34) (Figure 4C).

The metatarsals were processed for histological analysis by dehydration and hard acrylic embedding using standard histology techniques. Sections were made along the transverse (mediolateral) plane to include the fracture callus and adjacent diaphyseal bone. The sections were stained 
with Sanderson's Rapid Bone Stain and counter stained with Van Gieson. High-resolution images were obtained using a microscope (E800; Nikon; Tokyo, Japan), Spot Imaging System (Diagnostic Instruments; Sterling Heights, MI), and histomorphometry software (Image Pro; Media Cybernetics; Rockville, MD, USA). Ten-times magnification images were acquired and assembled into a composite image to perform static histomorphometry analyses of the stained sections in two ROIs: periosteal callus and endosteal callus. The periosteal callus ROI was defined with the mediolateral boundary being the region exterior to the bone surface contralateral to the orthopaedic plate, and proximodistal boundaries being the screw holes adjacent to the fracture site. The boundaries of the endosteal ROI were defined by the mediolateral edges of the diaphyseal cortical shell and the proximodistal edges of the fracture gap (Figure $4 D$ ). Fractional areas (\%) of fibrocartilagenous and mineralized tissues were calculated in each ROI.

\section{Statistical analysis}

All statistical analyses were performed using Minitab 18 (Minitab LLC; State College, PA, USA). Statistical comparison of in vitro antenna sensitivities was performed using a one-way analysis of variance (ANOVA) with significance between groups determined by post-hoc pairwise Tukey comparisons $(\alpha=0.05)$. The effects of fracture model on DEC antenna sensitivity rate of change, $\mu \mathrm{CT}$, and histomorphometry analyses were compared using two sample $t$-test comparisons $(\alpha=0.05)$, where $\mu \mathrm{CT}$ and histologic comparisons were made while blocking data to control for ROI. Postmortem biomechanical data comparisons were compared by one-way ANOVA.

\section{Results}

\section{DEC antenna sensitivity to orthopaedic implant deflections}

To evaluate the efficacy of dipole antennas to detect orthopaedic implant deflections, a stainless steel plate was introduced at various locations along the length of a linear dipole antenna while measuring the resultant shift in ARF apparent for all odd frequency harmonics (Figure 1A). For all resonant frequency harmonics tested, the quantity of antenna locations of maximum sensitivity to implant deflections increased with increasing harmonic number (Figure 1B). Relative DEC antenna sensitivity (S; \%) as a function of position from the end of the antenna (x; $\mathrm{mm})$, total antenna length $(\mathrm{L} ; \mathrm{mm})$, and the measurement harmonic number $(\mathrm{H}, \#)$ followed a quantifiable pattern (Eq. [1]); which was used to predict locations of maximum antenna sensitivity for higher order harmonics $(H>11)$ used in subsequent antenna designs.

$$
S(x, H)=0.5 *\left[1+\cos \left(\frac{\pi x(2 H-1)}{2 L}\right)\right]
$$

Using the above relationship, a three antenna array was created with the all antennas designed for measurement at the $25^{\text {th }}$ harmonic. This design was selected according to observations that DEC measurements at this harmonic produced minimal cross-talk between adjacent antennas. For each antenna, a linear dipole antenna was coiled to align three identified locations of maximum antenna sensitivity (51), with the goal of amplifying overall antenna sensitivity to implant deflections in a focused spatial region (Figure 2A).

\section{DEC antenna array sensitivity to cross-talk}

In using linear actuators to displace a stainless steel plate from the proximal antenna, while positioning identical stainless steel plates in static adjacency to the midspan and distal antennas (Figure $2 A$ ), the proximal antenna experienced a $1.081 \mathrm{MHz} \mathrm{ARF}$ shift while the midspan and distal antennas ARF shift magnitudes were $\leq 0.051 \mathrm{MHz}$ (Figure $5 A$ ). Similar analysis at the midspan antenna produced a $2.110 \mathrm{MHz}$ ARF shift at the midspan antenna, and ARF shift magnitudes at the adjacent antennas were $\leq 0.010 \mathrm{MHz}$ (Figure 5B). When this test was performed with the distal antenna, a $0.541 \mathrm{MHz}$ ARF shift was produced at the distal antenna, and the other antennas experienced ARF shift magnitudes of $\leq 0.067 \mathrm{MHz}$ (Figure 5C). These data demonstrated that three coiled antennas could be used in close proximity ( $\geq 4 \mathrm{~cm}$ ) to each other with minimal effect on adjacent antennas' ARF (i.e., antenna cross-talk) as a function of implant displacement.

\section{DEC antenna array sensitivity to orthopaedic plates}

To evaluate the sensitivity of the DEC antenna array to changes in antenna-implant distance in the presence of biological tissue, a LCP was covered in cadaveric ovine tissue and displaced from the three antenna array via a precision linear actuator (Figure $2 B$ ). The sensitivity of each antenna to displacement, defined as the instantaneous slope of the ARF shift/LCP-antenna distance curve (Figure 5D), 

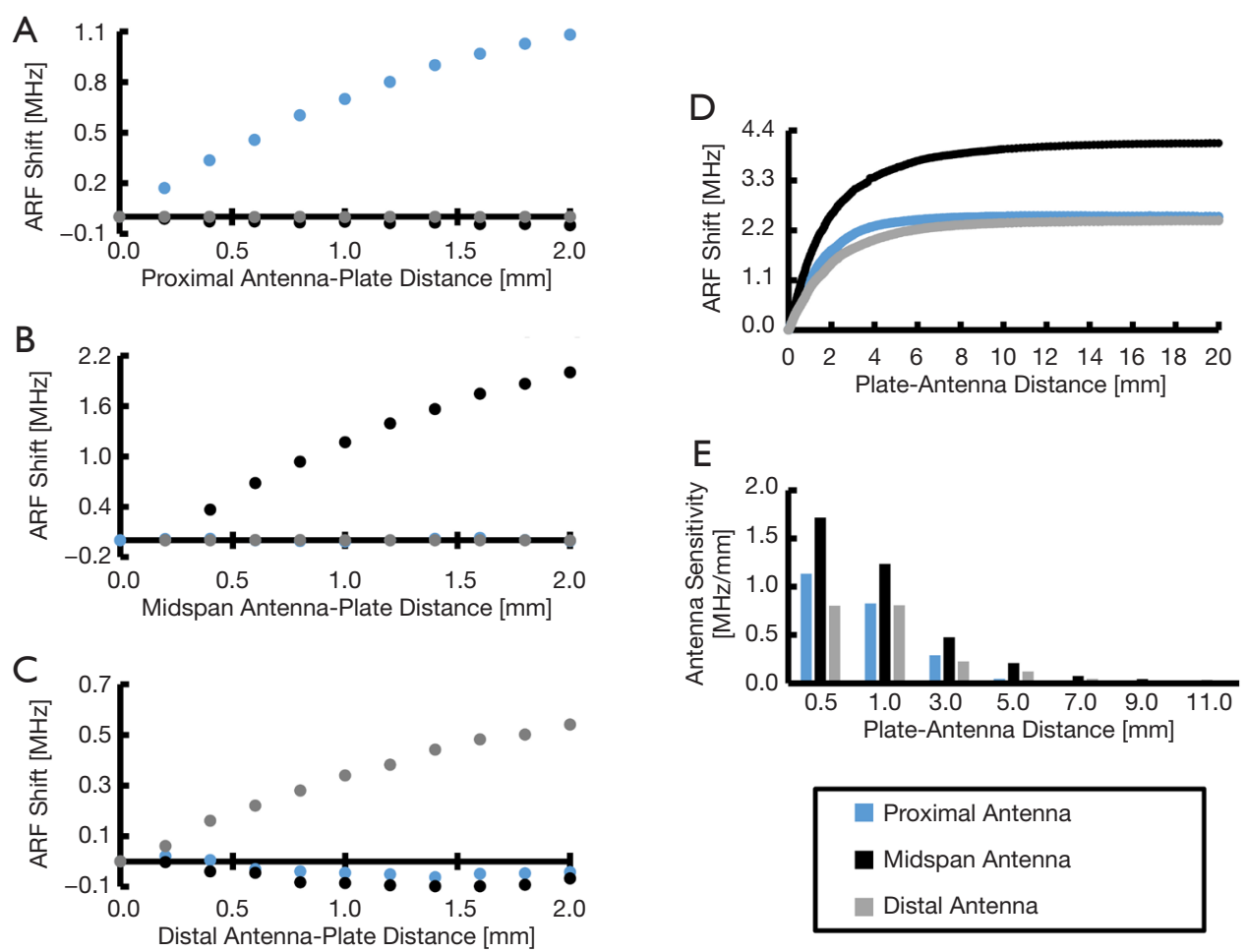

Figure 5 Comparative fracture model comparison. (A-C) Results of three-actuator antenna cross-talk experiments (methods depicted in Figure 2A). Graphs correspond to individually displacing the top, middle, or lower plate segments (A-C, respectively) while maintaining constant positioning of the other plate segments. Results illustrate maximum apparent resonant frequency (ARF) shifts at the site of the active linear actuator (i.e., the individual plate segment which was displaced from the antenna array) while ARF shifts remained less than 0.1 $\mathrm{MHz}$ for antennas away from the active linear actuator. (D) ARF shifts resulting from increasing distance between direct electromagnetic coupling (DEC) antennas and orthopaedic fracture plate covered in cadaveric ovine tissues (methods depicted in Figure 2B). (E) Instantaneous slope of each curve in (D) produces the antenna sensitivity at a given plate-antenna displacement.

decreased with increasing distance between the skin surface and antenna array. In increasing plate-antenna distance from 0.5 to $5.0 \mathrm{~mm}$, the proximal, midspan, and distal antenna sensitivities decreased by $96 \%, 88 \%$, and $85 \%$; respectively (Figure 5E).

\section{DEC differences in cadaveric fractures of varying stiffness}

To clarify the sensitivity of the developed DEC antenna array to changes in fracture site stiffness, fracture healing was simulated by progressively reducing the middiaphyseal stiffness of cadaveric ovine metatarsals treated by 9-hole LCP (Figure 3A). For each fracture state, fourpoint bending was applied to the metatarsal to produce physiologically relevant LCP deflections which were simultaneously measured by the DEC antenna array. A characteristic plot of antenna sensitivity as a function of location and fracture state for a single representative sample is provided in Figure 6. For the $2 \mathrm{CO}$ fracture state, all samples exhibited significantly different antenna sensitivities at all antenna locations. For all samples, the antenna sensitivity at the fracture site was significantly greater for the $2 \mathrm{CO}$ state than the $1 \mathrm{CO}$ and intact states, and the $1 \mathrm{CO}$ state was significantly more sensitive than the intact state for the majority of samples (6 of 8 samples). At locations away from the fracture site (i.e., the proximal and distal antennas), the majority of samples exhibited significantly greater antenna sensitivity for the $2 \mathrm{CO}$ state than the $1 \mathrm{CO}$ and intact states (7 of 8 samples).

\section{In vivo DEC temporal progression of proper and aberrant bealing fractures}

To better understand the clinical performance of DEC as 


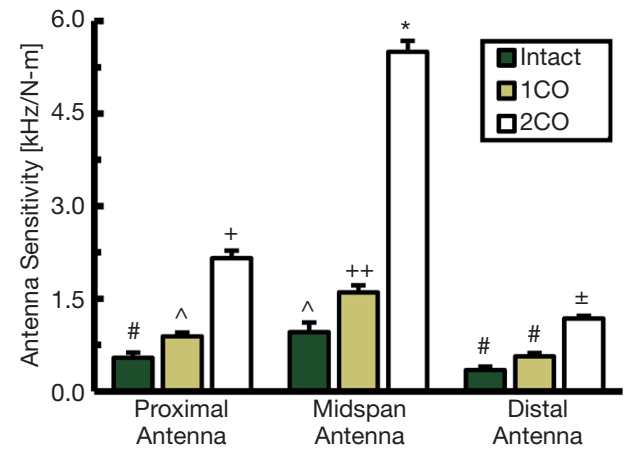

Figure 6 Mean direct electromagnetic coupling (DEC) antenna sensitivities (slope of apparent resonant frequency (ARF) shift per applied moment) for each antenna location and fracture state for a single representative cadaveric metatarsal sample. Intact, $1 \mathrm{CO}$, and $2 \mathrm{CO}$ fracture states respectively represent a progressive destabilization of the fracture site by reducing the quantity of cortical bone, as depicted in Figure $3 \mathrm{~A}$. Means with different symbols (i.e., ${ }^{*},+,++, \#, \pm$, or $\left.{ }^{\wedge}\right)$ are significantly different $(\mathrm{P}<0.05)$.

a diagnostic tool, an ovine comparative fracture model was implemented in which animals received a mid-diaphyseal metatarsal surgical osteotomy of large $(5.95 \pm 1.32 \mathrm{~mm})$ or small $(2.80 \pm 1.15 \mathrm{~mm})$ heights in order to produce delayed (DU) or proper (PU) healing outcomes, respectively. All fractures were treated by 9-hole LCP and weekly DEC diagnostic data was collected during four-point bending of the treated limb. The mean rate of change in DEC antenna sensitivity during early healing ( $<30$ days post-fracture) was significantly different between the DU $(-3.04 \pm 0.67$ $\% /$ day $)$ and $\mathrm{PU}$ fracture groups $(0.93 \pm 0.80 \% /$ day, $\mathrm{P}<0.001$, Figure $7 A$ ).

Fracture callus stiffness was quantified by servohydraulic four-point bending during post-mortem analysis. Cranial-caudal bending stiffness of the DU group $(n=4)$ was measured to be $10.77 \pm 7.58 \mathrm{~N}-\mathrm{m} / \mathrm{mm}$ and $3.62 \pm 1.59$ $\mathrm{N}-\mathrm{m} / \mathrm{mm}$ when subjected to four-point bending with orthopaedic hardware attached and removed, respectively. Similar analysis of the PU group $(n=3)$ produced bending stiffness of $15.66 \pm 2.00 \mathrm{~N}-\mathrm{m} / \mathrm{mm}$ and $14.15 \pm 4.04 \mathrm{~N}-\mathrm{m} / \mathrm{mm}$, respectively. One PU sample was omitted from biomechanical analysis due to experimental error. The bending stiffness of the intact contralateral metatarsi were $13.23 \pm 1.04 \mathrm{~N}-\mathrm{m} / \mathrm{mm}$ (Figure $7 B$ ).

For the DU fracture model, all animals exhibited maximum midspan antenna sensitivity within the first two weeks post-surgery (mean $8.75 \pm 3.50$ days post-surgery),

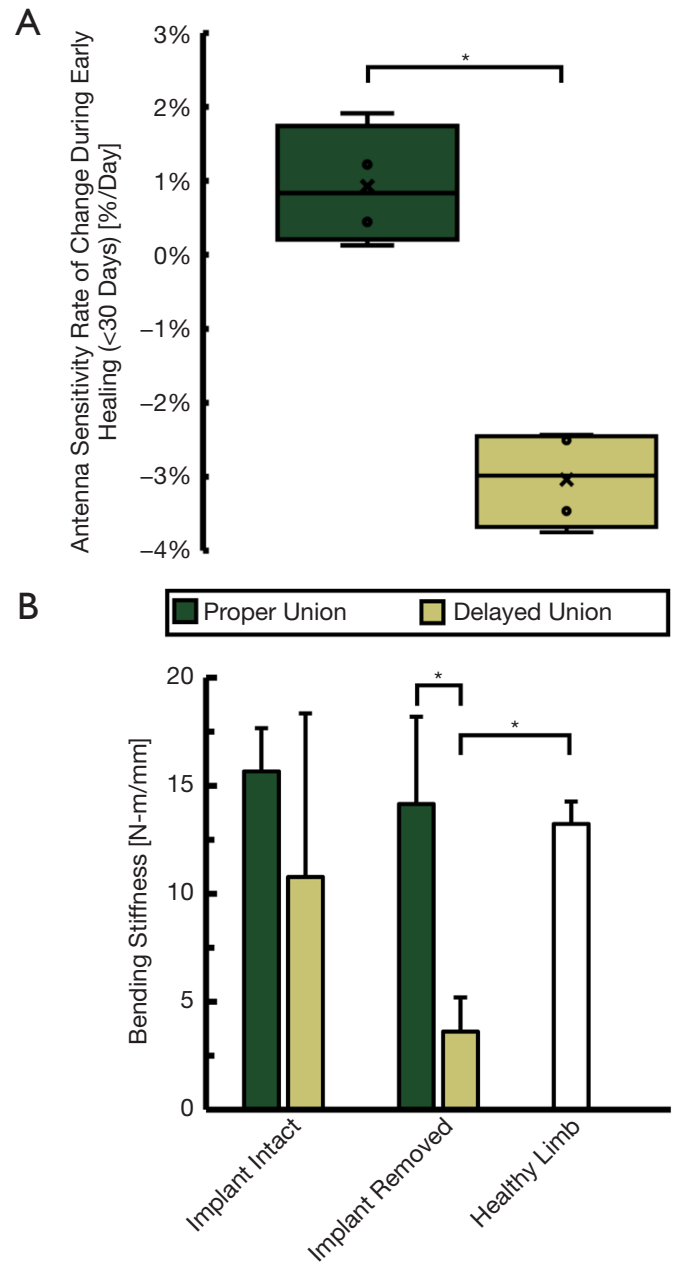

Figure 7 Direct electromagnetic coupling (DEC) diagnostic data and postmortem biomechanics comparison for in vivo fracture model. (A) Compiled results of DEC antenna sensitivity rate of change during early fracture healing in DEC diagnostic data for fractures trending towards union versus delayed union. Significant differences $(\mathrm{P}<0.05)$ are indicated by a *. (B) Fracture site bending stiffness as determined by post-mortem four-point bending in a servo-hydraulic loading fixture. Significant differences $(\mathrm{P}<0.05)$ are indicated by a *. Fracture site bending stiffness (i.e., with the plate implant removed) was significantly lower for the delayed union fracture model than the proper union fracture model $(\mathrm{P}=0.002)$ and healthy contralateral metatarsals $(\mathrm{P}=0.003)$.

and tended to decrease until animal sacrifice (Figure 8). For all DU specimens, the normalized midspan antenna sensitivities remained below $50 \%$ after four weeks postsurgery. Normalized midspan antenna sensitivity for this group decreased by an average of $81.2 \% \pm 18.0 \%$ from 


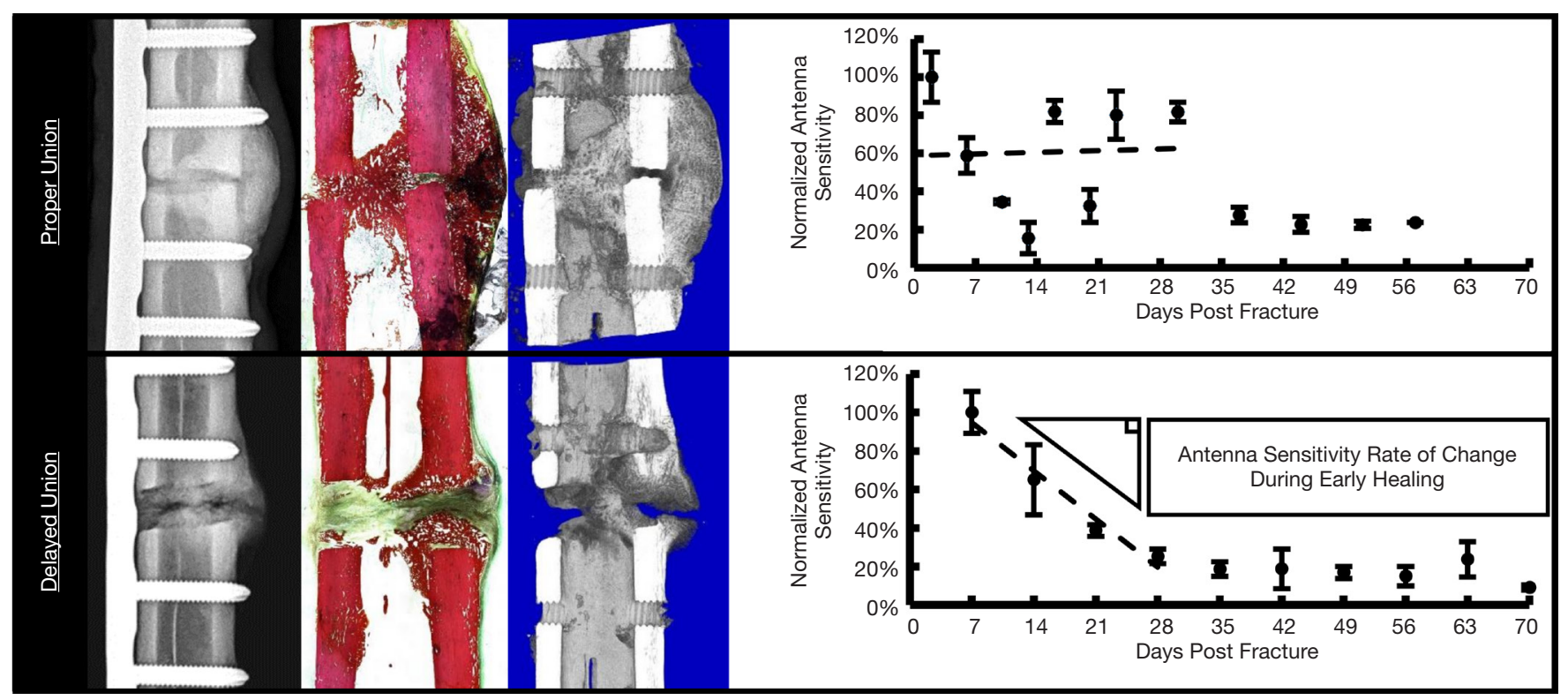

Figure 8 Comparison of in vivo fracture models. From left to right are X-ray images, mid-transverse histological section stained by with Sanderson's Rapid Bone Stain and counter stained with Van Gieson, mid-transverse micro computed tomography ( $\mu$ CT) image, and direct electromagnetic coupling (DEC) sensitivity measured at the fracture site throughout the study duration (mean \pm standard deviation of $\mathrm{n}=5$ replicate measurements per testing session). To account for inherent inter-specimen anatomical structural variations, antenna sensitivities are normalized by reporting as a percentage of the maximum observed sensitivity for each specimen during the study duration. Antenna sensitivity rate of change during early healing is calculated as the slope of a linear regression applied to antenna sensitivity data during the first 30 days of fracture healing.

maximum sensitivity to sensitivity at study's conclusion. The first radiographic indications of fracture site mineralization for the DU specimen occurred between weeks four and eight (Figure 9). Each specimen exhibited visual radiopacity at the fracture site, but had yet to achieve full cortical bridging; thus, it was concluded that all animals in this group exhibited a delayed fracture union healing pathway. The animals in the PU fracture model exhibited maximum midspan antenna sensitivity at $19.00 \pm 11.58$ days. Initial radiographic indications of fracture site mineralization occurred between three and four weeks post-surgery (Figure 9). All specimen in this group achieved clinical union based on successful cortical bridging at the animals' sacrifice.

TBV and BVF within both callus ROIs were on average larger for the PU fracture model than the DU fracture model, however these values were not statistically different (Figure 10A,10B). Periosteal callus and endosteal callus BMD were not significantly higher for animals of the PU fracture model $\left(504.5 \pm 37.8\right.$ and $357.8 \pm 109.6 \mathrm{mgHA} / \mathrm{cm}^{3}$, respectively) than the DU fracture model $(428.8 \pm 66.9$ and
$226.6 \pm 142.3 \mathrm{mgHA} / \mathrm{cm}^{3}$, respectively; Figure 10C).

According to postmortem histomorphometry analyses, mean callus area for the endosteal callus was not significantly different for the PU and DU fracture models $\left(155.0 \pm 39.4\right.$ and $104.8 \pm 82.0 \mathrm{~mm}^{2}$, respectively; Figure $10 D$ ), but were significantly different in the endosteal ROI $\left(25.7 \pm 7.8\right.$ and $65.8 \pm 21.8 \mathrm{~mm}^{2}$, respectively; Figure 10D). Additionally, the mean observed area fraction of bone within the periosteal callus and endosteal callus were significantly higher $(\mathrm{P}=0.009$ and $\mathrm{P}=0.003$, respectively) for animals of the PU model $(68.9 \% \pm 13.0 \%$ and $63.8 \% \pm 9.9 \%$, respectively) than the DU model $(32.1 \% \pm 12.2 \%$ and $31.6 \% \pm 6.4 \%$, respectively, Figure $10 E)$. Mean area fraction of fibrocartilage tissue was higher in both callus ROIs for the DU fracture group, but these differences were not statistically significant (Figure 10F).

\section{Discussion}

During DEC antenna design experiments, introduction of stainless steel to the linear coaxial cable antenna induced 


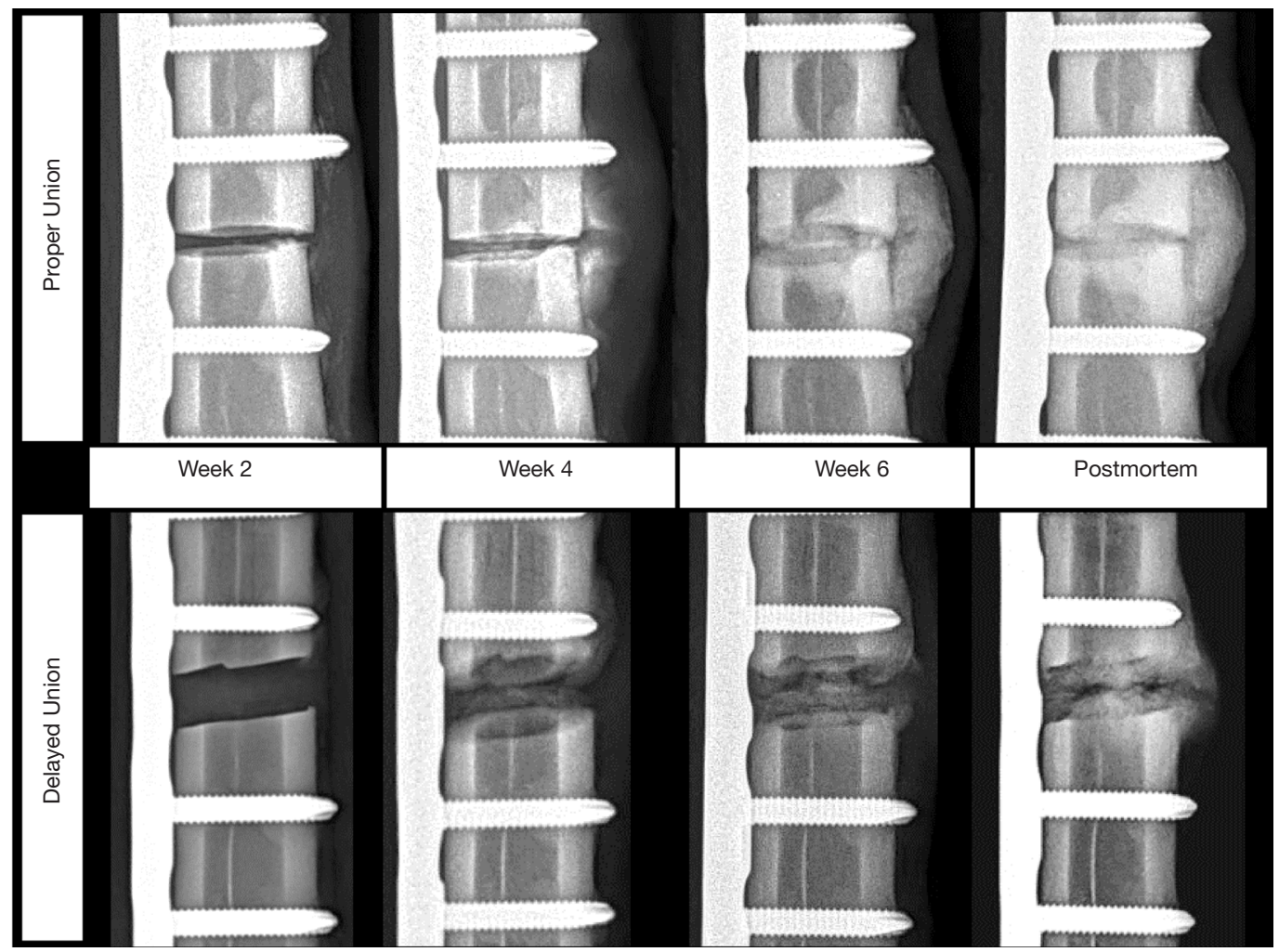

Figure 9 Radiographic progression of individual representative samples progressing towards proper (top row) or delayed (bottom row) fracture union.

large ARF shifts of some harmonics, while having little to no effect on the ARF of other harmonics. This supports the conclusion that this antenna type is effective at detecting the presence of orthopaedic plates, with the antenna sensitivity varying by location and resonant frequency harmonic number. Locations of maximum antenna sensitivity are of paramount importance for designing antennas to measure orthopaedic hardware deflection as these locations maximize the measurable ARF shift for small changes in antenna-plate displacement.

These results are consistent with the findings of Labus et al. where it was observed that coaxial cable DEC antennas exhibit increasing quantities of high sensitivity antenna locations as measurement harmonic number was increased (51). The results of the current study expand upon these findings by demonstrating this relationship to hold true for the first 11 ARF harmonics. Increasing harmonic number decreases the size of regions along the cable with high DEC sensitivity (Figure $1 B$ ). It may be advantageous to design DEC antennas for higher harmonics, therefore creating a more focused region of high sensitivity antenna measurements; however, increasing harmonic number results in elevated measurement frequency (i.e., shifting from $\mathrm{MHz}$ to $\mathrm{GHz}$ ) which is known to produce increased signal loss through biological tissues (54).

The multi-antenna array designed in this study exhibits minimal antenna ARF shifts when displacements are increased at an adjacent antenna (Figure 5A-5C), thus indicating minimal antenna cross-talk. Each antenna is therefore concluded to be independent in measuring their respective discrete spatial regions. However, it should be noted that this was measured using separate metal plates which were individually displaced. This is not directly representative of orthopaedic hardware deflections which are continuous over the length of the implant. Furthermore, translations of metal plates via linear actuators simulates rigid body displacements rather than bending induced deflections. These limitations were assumed to be permissible for initial antenna development given that more clinically relevant follow-up studies were performed, but care should nonetheless be taken when comparing these 


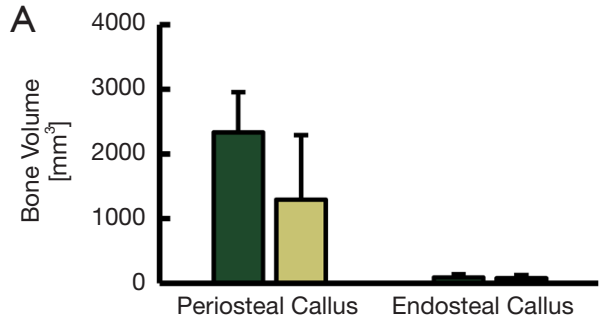

B

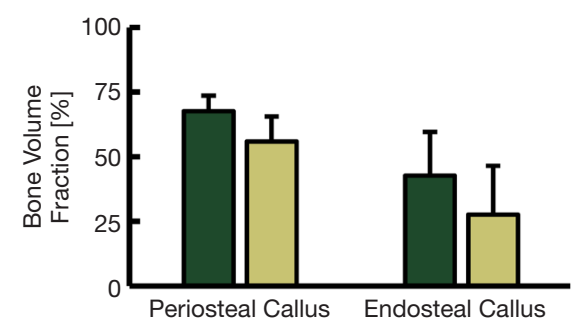

C

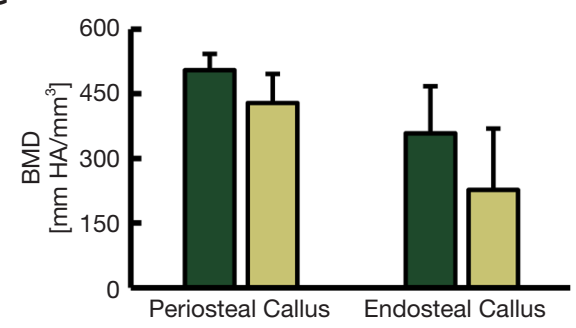

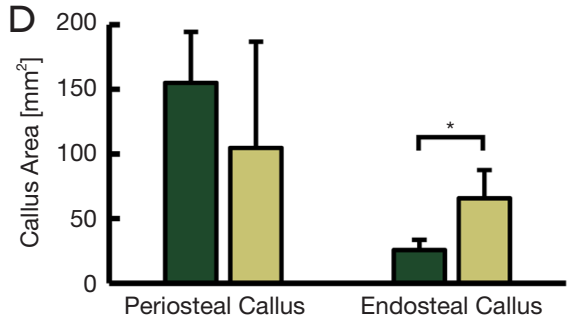

E

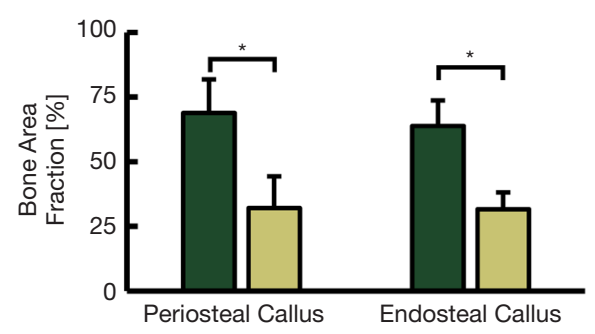

F

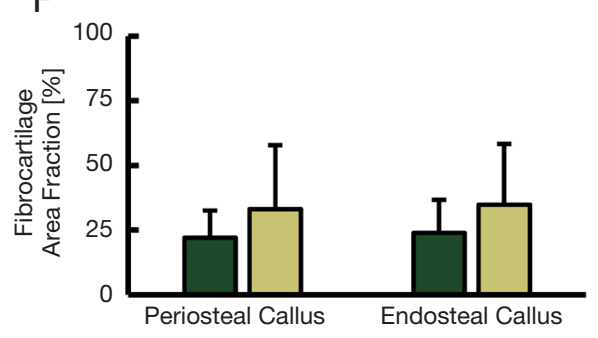

$\square$ Proper Union $\quad \square$ Delayed Union

Figure 10 Comparative fracture model postmortem micro computed tomography $(\mu \mathrm{CT})$ and histomorphometry. Significant differences $(\mathrm{P}<0.05)$ are indicated by $\mathrm{a}$ *. Mean results of $\mu \mathrm{CT}$ analysis of $(\mathrm{A})$ total bone volume $(\mathrm{TBV}),(\mathrm{B})$ bone volume fraction $(\mathrm{BVF}=\mathrm{TBV} /$ total volume), and (C) bone mineral density (BMD). For all analyses, there were no significant differences between proper union and delayed union models within either region of interest (ROI). (D-F) Mean histomorphometry results indicate total callus area to be significantly greater for the delayed union fracture model $(\mathrm{P}=0.04)$, and proper union bone area fraction to be significantly greater in both the periosteal and endosteal $(\mathrm{P}=0.009$ and $\mathrm{P}=0.003$, respectively).

benchtop data to in vitro and in vivo findings.

All antennas in the final array demonstrated the ability to produce quantifiable ARF shifts of greater than 2 $\mathrm{MHz}$ when increasing skin-antenna distance from 0 to $5 \mathrm{~mm}$ via linear actuator (Figure 5D), which supports the ability of this technique to measure mechanically induced orthopaedic hardware deflections in vivo. This test established differences in antenna sensitivity associated with antenna location, where the proximal and distal antennas exhibited $\geq 41.1 \%$ reduction in total ARF shifts relative to the middle antenna. Additionally, antenna sensitivity reduced appreciably with increasing distances of empty space between the antenna and LCP, as demonstrated by antenna sensitivity reducing by $\geq 85 \%$ when LCP-antenna distance exceeded $5 \mathrm{~mm}$ (Figure $5 E$ ). These data thus indicate that the current DEC antennas must be in close proximity to coupled members for measurement efficacy.

Use of an in vitro cadaveric fracture model introduced physiologic tissues and clinically relevant implant bending to provide a more translational approximation of DEC's performance in quantifying relative changes in fracture site stiffness. Antenna sensitivities were highly repeatable for 
a given fracture state (Figure 6) thus suggesting adequate implant-antenna coupling despite the introduction of physiologic tissues. The DEC antenna array further demonstrated the ability to quantify significant differences in antenna sensitivity associated with stiffness of the fracture site. Decreased occurrences of significant differences were observed at the antennas away from the fracture site, which can feasibly be attributed to a combination of reduced implant deflection at these locations and lower inherent antenna sensitivity. Based upon low data variance, nonlinear antenna sensitivity effects did not appear impactful within a given in vitro analysis of orthopaedic hardware deflection. This is consistent with the findings of Labus et al. (51) and can ostensibly be attributed to ARF shifts being approximately linear for the small scale deflections produced in this testing setup. However, non-linear effects may present challenges in comparing data from successive time points due to differences in initial implant-antenna distances resulting in changes to antenna sensitivity to hardware deflection.

Significant differences in antenna sensitivity were associated with antenna location within the array. In the case of simple mid-diaphyseal fractures, such as those simulated in this study, quantification of implant deflection away from the fracture site is of secondary interest. However, this information may prove expedient as a diagnostic tool for cases of non-diaphyseal or complex fracture types. Use of multi-location antenna arrays may be additionally useful for improving diagnostic accuracy by reducing data artifacts resulting from soft tissue compliance. DEC antennas measure relative changes to the implantantenna distance, but are indiscriminate to displacements resulting from mechanical deformation versus rigid body displacement. While the custom loading fixture used in this study was designed to minimize rigid body displacement of the fractured limb, these effects cannot be completely eliminated due to compression of compliant soft tissues (i.e., skin and muscle) at the application sites of bending loads. DEC antenna sensitivities are consequently amplified due to compression of soft tissues causing the entire limb to displace towards the antennas. Diagnostic DEC data relies upon relative changes in antenna sensitivities, rather than absolute values, thus rigid body effects are hypothesized to be inconsequential for clinical use of DEC provided that soft tissue compliance levels are consistent across testing sessions.

These rigid-body effects may be reduced nonetheless through use of a multi-location antenna array, rather than a single diagnostic antenna. In four-point bending, translation in the direction of bending at the stationary (outer) bending points is negligible for bending displacements alone. Thus, the multi-location DEC array may be used to measure deflections at these locations to quantify rigid body displacements, while simultaneously performing diagnostic DEC measurements at the fracture site. Subtracting these rigid body effects from the diagnostic measurements could then facilitate improved accuracy of measurements made at the fracture site. In theory, such a technique would obviate soft tissue compliance effects to provide an isolated measurement of relative implant stiffness which could not be obtained exclusively from four-point bending or singleDEC measurements.

For the in vivo fracture model, all animals of the PU group achieved successful fracture healing prior to study termination as supported by radiographic appearance of cortical bridging and postmortem bending stiffness values comparable to healthy untreated bone (Figure $7 B$ ). Conversely, terminal radiographic images of the DU fracture gap indicated failure to achieve cortical bridging. Radiographic callus presentation was varied in this treatment group, with three animals presenting large partially calcified endosteal and periosteal calluses, and one animal showing a complete lack of periosteal callus formation or calcification. The former case is indicative of mechanical instability at the fracture site and may heal if given excess time to heal; however, the latter case is suggestive of atrophic nonunion and is unlikely to heal without secondary clinical intervention (55-57). The radiographic indications of aberrant healing in this treatment group are supported by the postmortem biomechanics data where the observed mean fracture stiffness was significantly lower than healthy limbs or those of the PU treatment group (Figure 7B).

Despite the significant differences in observed fracture stiffness associated with surgical model, no significant differences were observed in $\mu \mathrm{CT}$ data. However, periosteal callus measurements of TBV, BVF, and BMD were trending towards being significantly greater in the $\mathrm{PU}$ model relative to the $\mathrm{DU}$ model $(\mathrm{P}=0.14, \mathrm{P}=0.095, \& \mathrm{P}=0.11$; respectively). These trends are supported by the observed histomorphometry data which indicated the PU model to express significantly higher bone area fractions in both the periosteal and endosteal callus regions (Figure 10E). These findings are consistent with previous studies which have demonstrated larger fracture gaps to experience delayed fracture healing (58) and reduced callus mineralization (59).

Differences between biomechanical and $\mu \mathrm{CT}$ analyses 
may stem from the inability of $\mu \mathrm{CT}$ to discern cortical bridging in the periosteal callus. For example, three samples in the DU fracture group exhibited hypertrophic periosteal callus formation in which large quantities of mineralized tissue were formed near the diaphyseal cortices, but very little formed at the fracture site. The resultant discontinuity of mineralized tissue across the fracture gap is expected to decrease fracture site mechanical stiffness while still exhibiting adequate levels of bone formation according to $\mu \mathrm{CT}$ analysis of the periosteal callus composition as a whole. This conclusion is supported by the histologic and $\mu \mathrm{CT}$ appearance of calcified tissue bridging of the fracture site in the PU group, which was accompanied by a significantly greater bending stiffness relative to the DU group (Figure $7 B$ ). These observations are consistent with the findings of Panjabi et al. (60) and Augat et al. (61) which established the importance of cortical continuity along the fracture line for restoring mechanical stability.

The biomechanical, histology, and $\mu \mathrm{CT}$ data suggest the two cohorts of this study to be a reasonable approximation of normal and aberrant/delayed fracture healing. In vivo radiographic evidence of callus mineralization was temporally adequate in the PU group (i.e., callus radiopacity appearing three to four weeks post-fracture) but was considerably postponed for the DU group (i.e., callus radiopacity appearing four to eight weeks post-fracture); thus, this study partially recapitulated the diagnostic deficits of $\mathrm{X}$-ray imaging for predicting fracture healing outcome. This is further supported by the appearance of radiopaque tissues at the fracture site in three of four DU animals despite reduced fracture stiffness (Figure $7 B$ ). These findings are consistent with current literature where it has been established that planar radiographic images are a poor predictor of stiffness (26) and strength (60) of the healing fracture.

While standard planar X-ray imaging was unable to identify changes to the osteotomy site during the early stages of fracture healing, DEC antenna diagnostics were able to detect significant differences between proper and delayed healing during the first 30 days of fracture healing (Figure 7A). Normalized DEC sensitivity from the DU group were greatest during the initial stages of healing (mean $8.75 \pm 3.50$ days post-surgery) followed by a steady decrease throughout the remaining study duration. The PU group exhibited observably different temporal DEC profiles throughout the study, and were generally characterized by a lack of temporal pattern (Figure 8). The present data agree with previous studies where it has been shown that fracture site stiffness may be indirectly quantified by monitoring fixation plate mechanics (32), and temporally repeated measurements can identify aberrant bone healing during early stages of healing $(31,40,62)$. DEC is advantageous for this application as it does not require direct measurement of implant strain, thus obviating the need for external fracture fixation or sensor instrumentation of custom internal fixation devices which has been associated with sensor failure (40) and difficulties in interrogating sensors through overlaying soft tissue (63).

While the significant differences observed within the first 30 days of healing marks a five-month improvement relative to mean nonunion diagnosis in humans (20), but care must be taken when extrapolating these results to human fractures due to inherent interspecies differences despite similarities in body weight, bone macrostructure (52), bone mineral composition, cortical bone microarchitecture, and bone healing rates (53). The current study also remains limited by small sample size and low variety of fracture type and treatment combinations. The possibility of unintentional bias cannot be excluded from these experiments due to non-blinded testing. As discussed in the proceeding text, this study is also limited by the lack of technical maturity inherent to any emerging technology including the DEC antenna used throughout these experiments.

Despite positive results for DEC in differentiating proper and aberrant healing pathways during initial stages of fracture repair, there remains a number of obstacles to be overcome for this diagnostic technique to achieve clinical utility. We hypothesize that the dissimilarity of the PU and DU fracture group antenna sensitivity profiles can be attributed to differences in fracture stiffness rate of change during initial fracture healing in combination with the data normalizing technique used in this study. It is thought that the DU group exhibits a gradual increase in fracture stiffness throughout the study duration while the PU group experiences a rapid improvement in fracture stiffness during the early stages of healing due to the smaller fracture gap and more expedient healing response. This theory aligns with results of previous studies where fractures exhibiting delayed healing present significantly reduced mineralized tissue formation and callus formation during the initial stages of healing, but later compensate through excess callus volume to achieve callus stiffness comparable to rapidly healing fractures (64). This is further supported by the expedited radiographic appearance of callus calcification in the PU group of the present study.

Based on this hypothesis, it is thought that the gradual changes in fracture stiffness of the DU group may be 
adequately quantified by weekly DEC measurements. In instances of rapidly stiffening fractures, however, DEC measurements would quickly become unable to reliably distinguish data noise from legitimate ARF shifts due to insufficient antenna sensitivity to exceedingly small plate displacements. Normalized DEC values would thus be similar from week to week as the antenna sensitivity is insufficient to detect further changes in plate displacement of the already stiff plate-fracture construct. This theory aligns with the present data as evidenced by the negative early healing rate of change in antenna sensitivity for the DU group $(-3.04 \pm 0.67 \% /$ day $)$ and the near zero value for the PU group $(0.93 \pm 0.80 \%$ /day, Figures $7 A, 8)$. These results emphasize the need for future technological developments to the DEC antennas to improve the sensitivity to small plate displacements. Such improvements will progress the clinical utility of this diagnostic by enabling better characterization and comparison of proper-, delayed-, and non-unions throughout the full duration of the healing timeline.

As previously discussed, the use of a multi-DEC antenna array is of particular interest for potential application towards averting DEC data artifacts resulting from soft tissue compliance. In using four-point bending to produce the implant deflections vital to DEC measurements, there is an unavoidable introduction of rigid body displacement of the treated limb resulting from compression of soft tissues at the limb-loading fixture contact points. These effects may potentially be mitigated through implementation of the multi-location DEC array to directly quantify limb rigid body displacements. However, the current design of the DEC antenna array is insufficient to enable measurements in a region of sufficiently discrete spatial resolution to facilitate such corrections; thus, future technological improvements will be pursued to this end.

Additional improvements for DEC must be centered upon real-time identification of aberrant fracture healing. While the current system was able to identify significant differences in healing pathway during the first 30 days postfracture, this result was obtained during post hoc analysis. For clinical utility, it is imperative that fracture healing outcome be identified within the initial stages of healing, rather than in retrospective analysis. The predictive ability of this technology may potentially be improved with additional insights into the mechanical progression of fractures from additional translational studies of larger populations with diverse fracture types. Furthermore, interpretation of early healing DEC diagnostic data would benefit from increased antenna sensitivity to facilitate improved characterization of the rapidly achieved fracture stiffness of properly healing fractures.

Future development of the DEC antenna and testing methods must also be focused on accounting for the nonlinear relationship between antenna-implant distance and DEC antenna sensitivities. Accounting for these effects, either through the development of an antenna with linear distance-sensitivity behavior or through calibration methods, would enable DEC measurements to provide a direct quantification of implant deflection. Doing so would reduce effects introduced by minor differences in limbantenna distance between testing session, and would provide clear insight to the exact mechanical state of the healing fracture site.

Further development of the DEC technology necessitates additional benchtop characterization of the effects of clinically pertinent variables on antenna sensitivity. For example, there exists a multitude of steel, cobalt-chromium, and titanium alloys which see pervasive use as orthopaedic implants, and implant alloy ostensibly affects DEC antenna sensitivity.

Furthermore, the cadaveric and in vivo tests performed in this study utilized a fracture model in which the LCP was covered by minimal amounts of overlaying skin, thus minimizing intervening tissues from impeding hardwareantenna coupling. However, many clinical fracture cases, such as treatment of femoral fractures, demand implantation of hardware below large depths of adipose and muscular tissues. The results of the current experiments indicate that DEC antenna sensitivity depreciates non-linearly with increasing antenna-skin surface distance (Figure $5 E$ ), but the impact of hard and soft tissue thickness is not known at this time. Future works must thus be executed to characterize these relationships to better inform the clinical viability of the current and subsequent antenna designs. Such information may advise whether antennas must be designed for lower frequency measurements where electromagnetic wave penetration through biological tissues is improved (54).

Despite the present limitations of this new technology, in vitro and in vivo data exhibit the efficacy of the DEC multi-antenna system for quantifying relative temporal changes in healing fracture stiffness. This technology demonstrated the ability to quantify differences in proper versus aberrant fracture healing at 30 days post-fracture. This marks a potential five-month improvement relative to average human clinical nonunion diagnosis times using X-ray imaging, although care must be taken when 
directly comparing the results of the present translational ovine fracture model to fracture healing in humans. In addition to its potential to accelerate diagnosis times to improve treatment of adverse fracture healing, DEC is advantageous as an emerging technology due to the relatively few regulatory hurdles associated with its clinical implementation. This can be attributed to its presumed compatibility with any existing metallic orthopaedic implant and its utilization of low energy radio frequency waves from a source external to the body. Thus, despite the need for ongoing technology maturation, DEC demonstrates potential as a clinical tool to expedite the diagnosis and treatment of failed bone fracture healing.

\section{Acknowledgments}

We thank Dr. Holly Stewart, Katie Bisazza, Cat Hersh, Lisa Mangin, Erin McCready, Meaghan Monahan, \& Izzy Olaes for their contributions to conducting in vivo biomechanics studies.

Funding: This project was supported by the National Institute of Health (NIH)-National Institute of Arthritis and Musculoskeletal and Skin Diseases (NIAMS) (R01AR069734-01-"Early Detection and Prediction of Complex Bone Fracture Healing”).

\section{Footnote}

Reporting Checklist: The authors have completed the ARRIVE reporting checklist. Available at https://dx.doi. org/10.21037/atm-21-1853

Data Sharing Statement: Available at https://dx.doi. org/10.21037/atm-21-1853

Conflicts of Interest: All authors have completed the ICMJE uniform disclosure form (available at https://dx.doi. org/10.21037/atm-21-1853). Funding for this project was supported by the NIH-NIAMS. Additional funding from the NIH and Colorado Office of Economic Development and International Trade (COEDIT) supported related research, but did not directly support this presented work. JGW and Drs. KML, CMP, and KCM are authors of patents related to the DEC technology presented in this manuscript. The other authors have no conflicts of interest to declare.

Ethical Statement: The authors are accountable for all aspects of the work in ensuring that questions related to the accuracy or integrity of any part of the work are appropriately investigated and resolved. Experiments performed by this study were approved by the Colorado State University Institutional Animal Care and Use Committee (CSU IACUC approval \#19-8990A), in compliance with United States and state of Colorado guidelines for the care and use of animals.

Open Access Statement: This is an Open Access article distributed in accordance with the Creative Commons Attribution-NonCommercial-NoDerivs 4.0 International License (CC BY-NC-ND 4.0), which permits the noncommercial replication and distribution of the article with the strict proviso that no changes or edits are made and the original work is properly cited (including links to both the formal publication through the relevant DOI and the license). See: https://creativecommons.org/licenses/by-nc-nd/4.0/.

\section{References}

1. Antonova E, Le TK, Burge R, et al. Tibia shaft fractures: costly burden of nonunions. BMC Musculoskelet Disord 2013;14:42.

2. Brinker MR, Hanus BD, Sen M, et al. The devastating effects of tibial nonunion on health-related quality of life. J Bone Joint Surg Am 2013;95:2170-6.

3. Heckman JD, Sarasohn-Kahn J. The economics of treating tibia fractures. The cost of delayed unions. Bull Hosp Jt Dis 1997;56:63-72.

4. Kanakaris NK, Giannoudis PV. The health economics of the treatment of long-bone non-unions. Injury 2007;38 Suppl 2:S77-84.

5. Claes L, Recknagel S, Ignatius A. Fracture healing under healthy and inflammatory conditions. Nat Rev Rheumatol 2012;8:133-43.

6. Oryan A, Monazzah S, Bigham-Sadegh A. Bone injury and fracture healing biology. Biomed Environ Sci 2015;28:57-71.

7. McKibbin B. The biology of fracture healing in long bones. J Bone Joint Surg Br 1978;60-B:150-62.

8. Zhang L, Miramini S, Richardson M, et al. The role of impairment of mesenchymal stem cell function in osteoporotic bone fracture healing. Australas Phys Eng Sci Med 2017;40:603-10.

9. Ghimire S, Miramini S, Richardson M, et al. Role of Dynamic Loading on Early Stage of Bone Fracture Healing. Ann Biomed Eng 2018;46:1768-84. 
10. Perren SM. Evolution of the internal fixation of long bone fractures. The scientific basis of biological internal fixation: choosing a new balance between stability and biology. J Bone Joint Surg Br 2002;84:1093-110.

11. Claes LE, Heigele CA. Magnitudes of local stress and strain along bony surfaces predict the course and type of fracture healing. J Biomech 1999;32:255-66.

12. Einhorn TA, Gerstenfeld LC. Fracture healing: mechanisms and interventions. Nat Rev Rheumatol 2015;11:45-54.

13. Marsh D. Concepts of fracture union, delayed union, and nonunion. Clin Orthop Relat Res 1998;(355 Suppl):S22-30.

14. Giannoudis PV, Kanakaris NK. Non-unions. In: Lasanianos NG, Kanakaris NK, Giannoudis PV, editors. Trauma and Orthopaedic Classifications: A Comprehensive Overview. London: Springer London, 2015:529-32.

15. Andrzejowski P, Giannoudis PV. The 'diamond concept' for long bone non-union management. J Orthop Traumatol 2019;20:21.

16. Giannoudis PV, Einhorn TA, Marsh D. Fracture healing: the diamond concept. Injury 2007;38 Suppl 4:S3-6.

17. Ghiasi MS, Chen JE, Rodriguez EK, et al. Computational modeling of human bone fracture healing affected by different conditions of initial healing stage. BMC Musculoskelet Disord 2019;20:562.

18. Zhang L, Richardson M, Mendis P. Role of chemical and mechanical stimuli in mediating bone fracture healing. Clin Exp Pharmacol Physiol 2012;39:706-10.

19. Gaston MS, Simpson AH. Inhibition of fracture healing. J Bone Joint Surg Br 2007;89:1553-60.

20. Bhandari M, Guyatt GH, Swiontkowski MF, et al. A lack of consensus in the assessment of fracture healing among orthopaedic surgeons. J Orthop Trauma 2002;16:562-6.

21. Whelan DB, Bhandari M, McKee MD, et al. Interobserver and intraobserver variation in the assessment of the healing of tibial fractures after intramedullary fixation. J Bone Joint Surg Br 2002;84:15-8.

22. Hak DJ, Fitzpatrick D, Bishop JA, et al. Delayed union and nonunions: epidemiology, clinical issues, and financial aspects. Injury 2014;45 Suppl 2:S3-7.

23. Nicholls PJ, Berg E, Bliven FE Jr, et al. X-ray diagnosis of healing fractures in rabbits. Clin Orthop Relat Res 1979;(142):234-6.

24. Blokhuis TJ, de Bruine JH, Bramer JA, et al. The reliability of plain radiography in experimental fracture healing. Skeletal Radiol 2001;30:151-6.

25. Hammer RR, Hammerby S, Lindholm B. Accuracy of radiologic assessment of tibial shaft fracture union in humans. Clin Orthop Relat Res 1985;(199):233-8.

26. McClelland D, Thomas PB, Bancroft G, et al. Fracture healing assessment comparing stiffness measurements using radiographs. Clin Orthop Relat Res 2007;457:214-9.

27. Davis BJ, Roberts PJ, Moorcroft CI, et al. Reliability of radiographs in defining union of internally fixed fractures. Injury 2004;35:557-61.

28. Claes L, Grass R, Schmickal T, et al. Monitoring and healing analysis of 100 tibial shaft fractures. Langenbecks Arch Surg 2002;387:146-52.

29. Webb J, Herling G, Gardner T, et al. Manual assessment of fracture stiffness. Injury 1996;27:319-20.

30. Hente R, Cordey J, Perren SM. In vivo measurement of bending stiffness in fracture healing. Biomed Eng Online 2003;2:8.

31. McGilvray KC, Unal E, Troyer KL, et al. Implantable microelectromechanical sensors for diagnostic monitoring and post-surgical prediction of bone fracture healing. J Orthop Res 2015;33:1439-46.

32. Stoffel K, Klaue K, Perren SM. Functional load of plates in fracture fixation in vivo and its correlate in bone healing. Injury 2000;31 Suppl 2:S-B37-50.

33. Wehner T, Gruchenberg K, Bindl R, et al. Temporal delimitation of the healing phases via monitoring of fracture callus stiffness in rats. J Orthop Res 2014;32:1589-95.

34. Gadomski BC, McGilvray KC, Easley JT, et al. Partial gravity unloading inhibits bone healing responses in a large animal model. J Biomech 2014;47:2836-42.

35. Grasa J, Gómez-Benito MJ, González-Torres LA, et al. Monitoring in vivo load transmission through an external fixator. Ann Biomed Eng 2010;38:605-12.

36. Claes LE, Cunningham JL. Monitoring the mechanical properties of healing bone. Clin Orthop Relat Res 2009;467:1964-71.

37. Ogrodnik PJ, Moorcroft CI, Thomas PB. Measuring multi-dimensional, time-dependent mechanical properties of a human tibial fracture using an automated system. Proc Inst Mech Eng H 2007;221:641-52.

38. Schmickal T, von Recum J, Wentzensen A. Stiffness measurement of the neocallus with the Fraktometer FM 100. Arch Orthop Trauma Surg 2005;125:653-9.

39. Seide K, Weinrich N, Wenzl ME, et al. Three-dimensional load measurements in an external fixator. J Biomech 2004;37:1361-9.

40. Seide K, Aljudaibi M, Weinrich N, et al. Telemetric assessment of bone healing with an instrumented 
internal fixator: a preliminary study. J Bone Joint Surg Br 2012;94:398-404.

41. Melik R, Perkgoz NK, Unal E, et al. Bio-implantable passive on-chip RF-MEMS strain sensing resonators for orthopaedic applications. J Micromech Microeng 2008; 18:115017.

42. Melik R, Unal E, Perkgoz NK, et al. Circular High-Q Resonating Isotropic Strain Sensors with Large Shift of Resonance Frequency under Stress. Sensors (Basel) 2009;9:9444-51.

43. Melik R, Unal E, Kosku Perkgoz N, et al. Flexible metamaterials for wireless strain sensing. Applied Physics Letters 2009;95:181105.

44. Melik R, Unal E, Perkgoz NK, et al. Metamaterialbased wireless strain sensors. Applied Physics Letters 2009;95:011106.

45. Melik R, Unal E, Perkgoz NK, et al. Metamaterial based telemetric strain sensing in different materials. Opt Express 2010;18:5000-7.

46. Melik R, Unal E, Perkgoz NK, et al. editors. Metamaterial-based wireless RF-MEMS strain sensors. 2010 IEEE Sensors, 2010 1-4 Nov. 2010.

47. Melik R, Unal E, Perkgoz NK, et al. RF-MEMS Load Sensors with Enhanced Q-factor and Sensitivity in a Suspended Architecture. Microelectron Eng 2011;88:247-53.

48. Melik R, Unal E, Perkgoz NK, et al. Nested Metamaterials for Wireless Strain Sensing. IEEE Journal of Selected Topics in Quantum Electronics 2010;16:450-8.

49. Wolynski JG, Sutherland CJ, Demir HV, et al. Utilizing Multiple BioMEMS Sensors to Monitor Orthopaedic Strain and Predict Bone Fracture Healing. J Orthop Res 2019;37:1873-80.

50. Labus KM, Sutherland C, Notaros BM, et al. Direct electromagnetic coupling for non-invasive measurements of stability in simulated fracture healing. J Orthop Res 2019;37:1164-71.

51. Labus KM, Notaros BM, Ilic MM, et al. A Coaxial Dipole Antenna for Passively Sensing Object Displacement and Deflection for Orthopaedic Applications. IEEE Access 2018;6:68184-94.

Cite this article as: Wolynski JG, Labus KM, Easley JT, Notaroš BM, Ilić MM, Puttlitz CM, McGilvray KC. Diagnostic prediction of ovine fracture healing outcomes via a novel multilocation direct electromagnetic coupling antenna. Ann Transl Med 2021;9(15):1223. doi: 10.21037/atm-21-1853
52. Pearce AI, Richards RG, Milz S, et al. Animal models for implant biomaterial research in bone: a review. Eur Cell Mater 2007;13:1-10.

53. Martini L, Fini M, Giavaresi G, et al. Sheep model in orthopedic research: a literature review. Comp Med 2001;51:292-9.

54. Johnson CC, Guy AW. Nonionizing electromagnetic wave effects in biological materials and systems. Proceedings of the IEEE 1972;60:692-718.

55. Phieffer LS, Goulet JA. Delayed unions of the tibia. J Bone Joint Surg Am 2006;88:206-16.

56. Bell A, Templeman D, Weinlein JC. Nonunion of the Femur and Tibia: An Update. Orthop Clin North Am 2016;47:365-75.

57. Thomas JD, Kehoe JL. Bone Nonunion. StatPearls. Treasure Island (FL): StatPearls Publishing Copyright (C) 2020, StatPearls Publishing LLC.; 2020.

58. Claes LE, Heigele CA, Neidlinger-Wilke C, et al. Effects of mechanical factors on the fracture healing process. Clin Orthop Relat Res 1998;(355 Suppl):S132-47.

59. Claes L, Eckert-Hübner K, Augat P. The fracture gap size influences the local vascularization and tissue differentiation in callus healing. Langenbecks Arch Surg 2003;388:316-22.

60. Panjabi MM, Walter SD, Karuda M, et al. Correlations of radiographic analysis of healing fractures with strength: a statistical analysis of experimental osteotomies. J Orthop Res 1985;3:212-8.

61. Augat P, Margevicius K, Simon J, et al. Local tissue properties in bone healing: influence of size and stability of the osteotomy gap. J Orthop Res 1998;16:475-81.

62. Kienast B, Kowald B, Seide K, et al. An electronically instrumented internal fixator for the assessment of bone healing. Bone Joint Res 2016;5:191-7.

63. Wilson DJ, Morgan RL, Hesselden KL, et al. A singlechannel telemetric intramedullary nail for in vivo measurement of fracture healing. J Orthop Trauma 2009;23:702-9.

64. Lienau J, Schell H, Duda GN, et al. Initial vascularization and tissue differentiation are influenced by fixation stability. J Orthop Res 2005;23:639-45. 